\title{
Lumbar Position Sense with Extreme Lumbar Angle
}

\author{
A. Maduri, M.S. ${ }^{1}$ and S. E. Wilson, Ph.D ${ }^{2}$ \\ ${ }^{1}$ National Institute for Occupational Safety and Health, Morgantown, WV \\ ${ }^{2}$ Department of Mechanical Engineering, University of Kansas, Lawrence, KS
}

\begin{abstract}
Tasks involving flexed torso postures have a high incidence of low back injuries. Changes in the ability to sense and adequately control low back motion may play a role in these injuries. Previous studies examining position sense errors of the lumbar spine with torso flexion found significant increases in error with flexion. However, there has been little research on the effect of lumbar angle. In this study, the aim of the study was to examine how position sense errors would change with torso flexion as a function of the target lumbar angle. Fifteen healthy volunteers were asked to assume three different lumbar angles (maximum, minimum and mid-range) at three different torso flexion angles. A reposition sense protocol was used to determine a subject's ability to reproduce the target lumbar angles. Reposition sense error was found to increase $69 \%$ with increased torso flexion for mid-range target curvatures. With increasing torso flexion, the increase in reposition sense errors suggests a reduction in sensation and control in the lumbar spine that may increase risk of injury. However, the reposition error was smaller at high torso flexion angles in the extreme target curvatures. Higher sensory feedback at extreme lumbar angles would be important in preventing over-extension or over-flexion. These results suggest that proprioceptive elements in structures engaged at limits (such as the ligaments and facet joints), may provide a role in sensing position at extreme lumbar angles. Sensory elements in the muscles crossing the joint may also provide increased feedback at the edges of the range of motion.
\end{abstract}

\section{Keywords}

spine biomechanics; lumbar; proprioception; motor control

\section{Introduction}

Manual materials handling tasks involving flexed torso postures have been associated with a high incidence of low back injuries [Marras et al., 1995]. Compressive loads on the lumbar spine are high in these flexed positions and may lead to injury [Granata et al., 2005; Marras et al., 1995]. However, Punnett et al. demonstrated that even when compressive loads are at levels considered safe by National Institute for Occupational Safety and Health (NIOSH) guidelines, many injuries occur [Punnett et al., 1991]. Research on spine dynamics has proposed that dynamic spinal stability may also play a role in low back injuries [McGill, 2001; Panjabi,

\section{(C) 2009 Elsevier Ltd. All rights reserved.}

Please address all correspondence to: S.E. Wilson, Ph.D. Human Motion Control Laboratory Department of Mechanical Eng., Univ. of Kansas 1530 W. $5^{\text {th }}$ Street, Lawrence, KS 66045 Phone: (785) 864-2103; FAX: (785) 864-5445 sewilson@ku.edu.

Publisher's Disclaimer: This is a PDF file of an unedited manuscript that has been accepted for publication. As a service to our customers we are providing this early version of the manuscript. The manuscript will undergo copyediting, typesetting, and review of the resulting proof before it is published in its final citable form. Please note that during the production process errorsmaybe discovered which could affect the content, and all legal disclaimers that apply to the journal pertain. 
2003]. In order to coordinate movement and maintain spinal stability, appropriate sensing of lumbar spine motion is essential [Panjabi, 1992a, 1992b; Solomonow et al., 1998]. It is therefore important to investigate the sensory system in the low back and how risk factors such as flexed torso postures may affect sensory function. Previous research has demonstrated that errors in lumbar spine position sense are increased in flexed torso postures [Gade and Wilson, 2007; Wilson and Granata, 2003]. This would suggest a loss in appropriate proprioception with torso flexion that should be investigated further.

The coordination of lumbar motion during torso flexion is a complex task. Highly flexed tasks have been associated with highly kyphotic lumbar angles [McGill and Kippers, 1994;

Solomonow et al., 2003]. While lumbar angle becomes more kyphotic with torso flexion, there is a range of potential lumbar angles possible for any given torso flexion angle (Figure 1). Lumbar-pelvic coordination (the lumbar angle assumed in a lifting task as a function of torso flexion) has been shown to vary with a number of factors including speed of motion and weight lifted [Commissaris et al., 2002; Granata and Sanford, 2000; Lariviere et al., 2000; McGorry and Hsiang, 2000]. As a person flexes their trunk, a range of lumbar angles (more lordotic to more kyphotic) can be assumed at most torso postures.

The range of potential lumbar angles easily achievable at any given torso flexion can be thought of as a lumbar range of motion. At the end of this range, tension in the spinal ligaments and/ or compression in the facet joints could limit further motion [Panjabi, 1992a, 1992b]. It is thought that elements in the ligamentous tissues provide sensory information at end ranges of motion outside the neutral zone [Panjabi, 1992a, 1992b; Solomonow et al., 2003]. While engagement of these tissues and their sensory role has been demonstrated at extreme torso flexion angles, little evidence exists for the role of these tissues during more moderate torso flexion angles [Solomonow et al., 2003].

In addition to the passive tissues of the spine, sensory elements in the muscle, such as the muscle spindle organs, can also contribute to proprioception [Brumagne et al., 2000]. As a subject moves to the limits of the range of motion, changing lines of action of these muscles may also alter the ability of the muscle spindle organs to sense lumbar posture. It is, therefore, important to examine how sense of lumbar posture is altered by lumbar angle.

The aim of the present study was to assess the ability to sense lumbar angle as a function of both lumbar angle and torso flexion in order to understand how lumbar proprioception changes in flexion tasks. It was hypothesized that reposition sense error in the lumbar spine will increase with an increase in torso flexion, as previously observed, but will decrease at the extremes of lumbar angle due to additional feedback from stretched spinal ligaments and/or compressed facet joints.

\section{Methods}

Nine men and six women participated in this study. Subjects had a mean height of 172.07 (SD 11.73 ) $\mathrm{cm}$, a mean weight of 75.2 (SD 13.1) kg, and ages ranging from 21-34 years (mean 24.53 , SD $3.38 \mathrm{yrs}$ ). This study was approved by the human subjects committee of the University of Kansas and consent was obtained from all subjects. All the participants were healthy and reported no instance of low back pain within the last year or musculoskeletal disorder that would limit normal torso flexion.

An electromagnetic motion analysis system (Motion Star, Ascension Tech., VT) was used to collect position and orientation of three electromagnetic sensors. This system has a resolution of $0.08 \mathrm{~cm}$ and 0.1 degrees and an RMS accuracy of $0.76 \mathrm{~cm}$ and 0.5 degrees. The rotational accuracy was further validated in the laboratory by connecting one electromagnetic sensor to a $100 \mathrm{Kohms}$ rotational potentiometer, rotating the sensor and potentiometer through 120 
degrees of rotation at 5 degree intervals, and calculating the RMS error between the sensor and potentiometer.

The three sensors were attached to the skin with double-sided tape over the T10 spinous process, over the S1 spinous process, and over the manubrium. This kinematic data was collected for 5 seconds at a frequency of $30 \mathrm{~Hz}$ for each trial. Using the position of the T10 and $\mathrm{S} 1$ sensors, trunk flexion angle was determined as the angle between a line connecting these sensors and vertical. The difference in angular orientation between the T10 and S1 sensors in the anterior-posterior plane was defined as the lumbar angle. The manubrium marker allowed detection of trunk rotation and asymmetry of motion. This configuration is consistent with previous literature on lumbar position sense and lumbar-pelvic coordination [Gade and Wilson, 2007; Granata and Sanford, 2000; Wilson and Granata, 2003].

Before conducting the experimental protocol, the range of lumbar angle for the three flexion angles ( 0,30 and 60 degrees) was determined. A real-time visual display of torso flexion and lumbar angle was provided to the subjects (Figure 2). At each of the three torso flexion angles, subjects were instructed to hold the flexion angle constant while rotating the thorax and pelvis to change the lumbar angle. Subjects were allowed to practice these movements until they became comfortable with the equipment. Subjects were then instructed to assume the maximum (kyphotic) and then the minimum (lordotic) lumbar angle while maintaining one of the three torso flexion angles. Subjects were asked to repeat these extreme lumbar angles three times at each of the three torso flexion angles. The mean of the three maximum lumbar angles and the mean of the three minimum lumbar angles at each torso flexion angle were recorded. These means were used to define the three target lumbar angles: a maximum lumbar angle, a minimum lumbar angle and a mid-range lumbar angle (midpoint between the maximum and minimum lumbar angle) for each torso flexion angle.

A reposition sense protocol was then used to determine the subject's ability to sense and to reproduce the three target lumbar angles at the three flexion angles $(0,30$, and 60 degrees). The reposition sense protocol consisted of 8 trials. In the initial training trial, subjects were asked to assume a target position by matching both prescribed torso flexion and the target lumbar angle using real-time visual feedback of torso flexion and lumbar angle provided on the computer screen (Figure 2). The subjects were not told where the target lumbar angle was located within their range and were instructed only to "match the target angle displayed". After matching both the target flexion and lumbar angle, subjects were asked to remain in that posture while kinematic data was collected for 5 seconds. Subjects were instructed to remember this lumbar angle. The training trials were repeated three times with a short flexion task in between each trial.

After the initial three training trials, an assessment trial was performed. In the assessment trial, the lumbar angle display was turned off while flexion display remained available to the subject. While maintaining the specified torso flexion, subjects were asked to reproduce the target lumbar angle from memory and data was again collected for 5 seconds. Training and assessment trials were then alternated for a total of 8 trials: 5 training and 3 assessment trials. In between each trial, subjects were asked to return to upright standing and perform a torso flexion task to prevent holding of the lumbar angle.

Each subject performed the reposition sense protocol at all combinations of three target lumbar angles (maximum, minimum and mid-range lumbar angle) and three flexion angles $(0,30, \&$ 60 degrees) for a total of 9 separate tests. The order in which the tests were performed was randomized and subjects were asked to rest for 5 minutes before performing the next reposition sense protocol. 
Reposition sense error (RSE) was defined as the difference (in degrees) between the target lumbar angles $\left(\theta_{t}\right)$ and actual lumbar angle reproduced by the subject $\left(\theta_{\mathrm{r}}\right)$. The absolute value of this error (Absolute Reposition Sense Error (aRSE)) for each trial was used to determine the magnitude of the error:

Absolute Reposition Sense Error $(a R S E)=\left|\left(\theta_{\mathrm{t}}\right)-\left(\theta_{\mathrm{r}}\right)\right| \quad$ Eq. 1

Directional reposition sense error (Directional Reposition Sense Error (dRSE)) was defined as the difference between the target lumbar angles $\left(\theta_{t}\right)$ and reproduced (mean) lumbar angle $\left(\theta_{\mathrm{r}}\right)$ :

Directional Reposition Sense Error $($ dRSE $)=\left(\theta_{\mathrm{t}}\right)-\left(\theta_{\mathrm{r}}\right)$ Eq. 2

The absolute reposition sense errors and the directional reposition sense errors were averaged for the 5 training trials and for the 3 assessment trials at each of the nine experimental conditions. The repositioning errors for all nine conditions (three lumbar angle targets and three flexion angles) were compared to study the effect of torso flexion and lumbar angle on the reposition sense of the lumbar spine.

A Huynh-Feldt, repeated measures ANOVA was used to the examine the effects of the independent variables, torso flexion angle and lumbar angle, on the dependent variable, assessment absolute reposition sense error (aRSEa). Additional ANOVA were performed with the dependent variables, training absolute reposition sense error (aRSEt), training directional reposition sense error (dRSEt), and assessment directional reposition sense error (dRSEa). These tests were determined to be significant for a $\mathrm{p}<0.05$.

The significant main effects from the ANOVAs on the absolute reposition sense error (aRSEa) were further investigated using one-way, Huynh-Feldt repeated measures ANOVA performed to assess the effect of flexion on aRSEa for each lumbar angle condition. This analysis was done to confirm that absolute reposition error increased with torso flexion in mid-range curvatures as reported in the literature [Wilson and Granata, 2003]. One-way, Huynh-Feldt repeated measures ANOVA were also performed to assess the effect of lumbar angle target on aRSEa at each of the three torso flexion angles.

\section{Results}

Before the position sense protocols, three maximum and three minimum lumbar angles were obtained for each of the three flexion angles (Table 1). For the maximum lumbar angles, the average subject had a range of 4.4 degrees at 0 degrees of flexion, 5.3 degrees at 30 degrees of flexion, and 4.2 degrees at 60 degrees of flexion between their lowest and highest maximum lumbar angle. For the minimum lumbar angles, the average subject had a range of 9.6 degrees at 0 degrees of flexion, 6.3 degrees at 30 degrees of flexion and 8.3 degrees at 60 degrees of flexion. Compared to position sense errors (Table 2), these ranges were larger.

In this study, variation in absolute reposition sense error (aRSE) was assessed for both training (visual feedback available) and assessment (visual feedback removed) trials for all the target lumbar angles (maximum, minimum, and mid-range lumbar angles) at each torso flexion angle $\left(0^{0}, 30^{0}, \& 60^{0}\right)$. The directional reposition sense error (dRSE) was also assessed for all conditions to examine whether the errors in lumbar angle were biased in a particular direction. Because the angular accuracy of the electromagnetic sensors is important to the measurement of these errors, the RMS error was assessed in the laboratory setting against a rotational potentiometer. At each angle measured, the RMS error from the mean for the electromagnetic 
sensors was 0.06 degrees. Relative to the rotational potentiometer, an RMS error of 0.63 degrees was observed for measurements through 120 degrees of rotation.

The absolute reposition sense error for training trials (aRSEt) was small, averaging 1.15 (SD 1.24) degrees and was not significantly different with torso flexion, lumbar angle target, or the interaction of these variables (flexion, $p=0.176$, curvature, $p=0.962$, interaction, $p=0.622$, Table 2, Figure 3). The absolute reposition sense error for the assessment trials (aRSEa) averaged 3.59 (SD 2.49) degrees. A repeated-measures ANOVA of the assessment absolute reposition sense error (aRSEa) demonstrated significant changes in aRSEa with lumbar angle and with the interaction between lumbar angle and torso flexion ( $\mathrm{p}<0.05$, Table 3, Figure 3). To further clarify these interactions between torso flexion and lumbar angle, one-way ANOVAs were used to examine torso flexion with constant lumbar angle and lumbar angle with constant flexion. Absolute reposition sense error (aRSEa) was found to increase significantly with torso flexion for mid-range target lumbar angles (Table 4, Figure 3). aRSEa decreased significantly at both maximum and minimum target lumbar angles relative to the mid-range error at 60 degrees of torso flexion (Table 4, Figure 3). Only two subjects did not follow this pattern at 60 degrees of torso flexion. These results demonstrated that although the absolute reposition sense error (a measure of the overall magnitude of error) increased with torso flexion for mid-range lumbar angles, these errors were smaller with high torso flexion for extreme postures.

The directional reposition sense error (dRSE) was assessed to examine whether errors in lumbar angle were biased to one direction. The overall mean dRSE during training trials was close to zero ( -0.22 (SD 1.48)) and did not vary significantly with either torso flexion or lumbar angle (flexion, $p=0.110$, curvature, $p=0.478$, Table 5, Figure 4). During the assessment trials, $d R S E$ was found to be significantly altered ( $p<0.05$, Table 3 , Figure 4$)$ with target lumbar angle, with negative mean errors at maximal lumbar angle targets ( -0.96 (SD 2.28)) and positive mean errors at minimum lumbar angle targets (0.87 (SD 3.13)) (Table 5).

\section{Discussion}

In this study, the absolute reposition sense error was found to increase significantly with torso flexion in mid-range lumbar angles. With high torso flexion, the absolute reposition sense error was significantly smaller at the extreme lumbar angles than at mid-range lumbar angles. Wilson and Granata [2003] reported that absolute reposition sense error increased with torso flexion and suggested that poor control of lumbar angle may be a factor in the increased risk of low back pain with torso flexion [Wilson and Granata, 2003]. Gade and Wilson [2007] concluded that increase in reposition sense error of lumbar spine in flexed tasks is associated with torso flexion and not with changes in moment load [Gade and Wilson, 2007]. Gade and Wilson proposed that this increase in error may be due to altered alignment of trunk musculature reducing the ability of muscle spindle organs to sense lumbar angle. The results of this paper for the mid-range lumbar angle agree with these previous findings.

In this study, the range of lumbar angles possible at three different torso flexions was assessed and this range was used to create the target postures for a reposition sense protocol. The limits of this measured range could be a result of the characteristics of the passive structures of the spine (ligaments and facet joints for example), the resistance of the muscle/tendon units crossing the lumbar region of the torso or limits of strength in the torso musculature. Further research would be required to determine the relative roles of these limiting mechanisms. What can be said in this research is that this range of lumbar angles gives an estimate of where elastic resistance begins to limit rotation of the lumbar spine. From the range of lumbar angles a subject exhibited during the three measures of lumbar angle at any given condition, it can be observed that the limit of the range of motion is, in itself, not easy to sense (Table 1.B). This is further 
supported by the smaller numbers seen in position sense errors (Table 2) relative to those for the range of maximums and minimums. These smaller errors would suggest that targeting a position is more accurate than attempting to sense the limit of the range.

In extreme lumbar angles, the lumbar spine may be pushed to the kyphotic (in maximum lumbar angle) and lordotic (in minimum lumbar angle) limits of its range of motion. At maximum lumbar angle, one might expect compression of the anterior intervertebral disk as well as tension in the posterior ligaments and muscles including the supraspinous ligament, interspinous ligament, posterior longitudinal ligament, ligmentum flavum, and erector spinae muscles. At minimum lumbar angle, one might expect tension in the anterior longitudinal ligament, compression of the facet joints, and possibly tension in anterior trunk muscles such as the psoas. While the muscle spindle organs are thought to be the dominate sensory organ in joint proprioception [Brumagne et al., 1999], several authors have demonstrated the sensory roles of elements in the passive soft tissues of the spine [Khalsa et al., 2002; Roberts et al., 1995; Solomonow, 2004; Solomonow et al., 1998]. Solomonow et al. has demonstrated that tension in the supraspinous ligament can illicit reflex responses in the paraspinal musculature suggesting a sensory role for the spinal ligaments [Solomonow, 2004; Solomonow et al., 1998]. Khalsa et al. has demonstrated a similar role for the lumbar facet joints [Khalsa et al., 2002]. In this experiment, reduced absolute reposition sense error was observed at maximum lumbar angle giving support to the idea that stretch of the posterior spinal ligaments may play a role in sensing of lumbar angle. Reduced absolute reposition sense error observed at the minimum lumbar angle also gives credence to the potential role of the compression of the lumbar facet joints in sensing lumbar angle.

The directional reposition sense error during assessment was also found to be significantly related to lumbar angle. The directional reposition sense error is a measure of any bias in the error towards a particular direction (more or less lumbar angle). As might be expected, in the extreme lumbar angles, the errors were found to be directed towards the more neutral, midrange curvature. However, this bias was generally small and most of the $95 \%$ confidence intervals contained zero except for the maximum lumbar angle condition at 30 and 60 degrees.

In this study, the absolute reposition sense error for the training trials was small and the directional reposition sense error for the training trials was close to zero. These errors in training trials did not change significantly with torso flexion or lumbar angle target. The small training trial errors, particularly in the extreme lumbar angles, demonstrate that the required positions were feasible for the subjects and were not past physiological endpoints. During assessment, the absolute reposition sense error increased considerably with the loss of the visual confirmation of lumbar angle. This also confirms the experimental design by demonstrating that errors during the assessment trials were a function of proprioceptive abilities rather than subject training or equipment accuracy.

Overall, these results further support the results of Solomonow et al. and Khalsa et al., demonstrating the potential role of passive structures such as the spinal ligaments and facet joints in sense and therefore control of lumbar spine curvature at extreme lumbar angles [Khalsa et al., 2002; Solomonow, 2004; Solomonow et al., 1998]. This paper demonstrates that these extreme curvatures may occur not only at extreme torso flexion (typically greater than 90 degrees of torso flexion [Solomonow et al., 2003]) but also throughout the range of torso motion. The sensory mechanisms at these extreme lumbar angles can be thought of as the safety catch of the lumbar stabilization system. When the lumbar spine reaches the extremes of lumbar angle, the spinal ligaments, intervertebral discs and facet joints not only experience greater loading but could also be damaged by such loading. Such damage may not only result in low back pain but may also result in damage of the mechanotransducer elements in these tissues. In addition, McGill and Kippers [1994] and Solomonow et al. [2003] reported that spinal 
ligaments, when subjected to prolonged flexion positions, undergo a viscoelastic stretch resulting in decreased reflexive activity [McGill and Kippers, 1994; Olson et al., 2004;

Solomonow et al., 2003]. The proprioceptive contribution of the ligaments may be reduced by repeated exposure to extreme lumbar angles. This should be investigated further. Previous studies have also demonstrated increased position sense errors in subjects with a history of low back pain and injury [Brumagne et al., 2000; Gill and Callaghan, 1998; Koumantakis et al., 2002; Newcomer et al., 2000]. With injury and potential damage to the soft tissues of the lumbar spine, the decreased error in extreme curvatures demonstrated in this work may be altered. Future studies should examine how low back injury may alter the position sense in extreme lumbar angles.

In conclusion, the magnitude of the reposition sense error (aRSEa) increased with torso flexion in mid-range lumbar angles and decreased dramatically at extreme lumbar angles, particularly at high torso flexion angles. The increased error with flexion in mid-range curvatures agrees with previous studies by the investigators and suggests that appropriate sensing and therefore stability control of lumbar angle may be reduced in flexed working tasks. The decrease in the magnitude of error with extreme lumbar angles suggests that limit-sensing elements, possibly in the ligamentous tissues or facet joints, may play an important role in position sense and stability control in these extreme curvatures. While error is decreased in these curvatures suggesting better control, this control may be necessary due to increased loading of the passive structures of spine and potentially greater risk of injury. Future work should examine the potential changes in these behaviors with stretching of the ligamentous structures and with injury.

\section{Acknowledgements}

This work was supported in part by Whitaker Foundation Biomedical Engineering Research (Grant RG-03-0043) and by the BRIN Program of the National Center for Research Resources (NIH Grant Number P20 RR16475).

The findings and conclusions in this report are those of the author(s) and do not necessarily represent the views of the National Institute for Occupational Safety and Health.

\section{Biography}

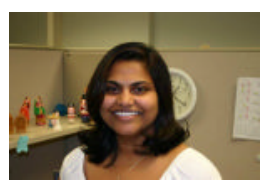

Anupama Maduri earned her M.S. in Mechanical Engineering from the University of Kansas in 2005. She is currently working as a Research Engineer at the National Institute for Occupational Safety and Health Laboratory in Morgantown, West Virginia. Her research interests are in spine biomechanics, modeling of work activities and integration of sensor technology.

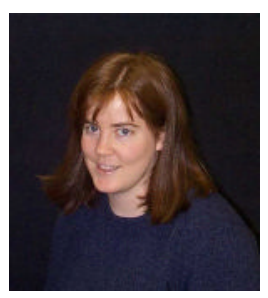

Sara E. Wilson earned a Ph.D. in Medical Engineering/ Medical Physics for Massachusetts Institute of Technology in 1999. After post-doctoral work at the University of Virginia, Dr. Wilson joined the faculty at the University of Kansas as an Assistant Professor in Mechanical 
Engineering. Her research interests are in spine biomechanics and the neuromotor control of lumbar motion.

\section{References}

Brumagne S, Cordo P, Lysens R, Verschueren S, Swinnen S. The role of paraspinal muscle spindles in lumbosacral position sense in individuals with and without low back pain. Spine 2000;25(8):989-994. [PubMed: 10767813]

Brumagne S, Lysens R, Swinnen S, Verschueren S. Effect of paraspinal muscle vibration on position sense of the lumbosacral spine. Spine 1999;24(13):1328-1331. [PubMed: 10404575]

Commissaris DA, Nilsson-Wikmar LB, Van Dieen JH, Hirschfeld H. Joint coordination during wholebody lifting in women with low back pain after pregnancy. Arch Phys Med Rehabil 2002;83(9):1279_ 1289. [PubMed: 12235609]

Gade VK, Wilson SE. Position sense in the lumbar spine with torso flexion and loading. J Appl Biomech 2007;23(2):93-102. [PubMed: 17603129]

Gill KP, Callaghan MJ. The measurement of lumbar proprioception in individuals with and without low back pain. Spine 1998;23(3):371-377. [PubMed: 9507628]

Granata KP, Lee PE, Franklin TC. Co-contraction recruitment and spinal load during isometric trunk flexion and extension. Clin Biomech (Bristol, Avon) 2005;20(10):1029-1037.

Granata KP, Sanford AS. Lumbar-pelvic coordination is influence by lifting task parameters. Spine 2000;25(11):1413-1418. [PubMed: 10828924]

Khalsa, PS.; Chiu, JB.; Aliberti, N.; Sileo, M. Biomechanical evidence for proprioceptive function of lumbar facet joint capsule.. 4th World Congress on Biomechanics; Calgary, CA. 2002.

Koumantakis GA, Winstanley J, Oldham JA. Thoracolumbar proprioception in individuals with and without low back pain: Intratester reliability, clinical applicability, and validity. Journal of Orthopaedic \& Sports Physical Therapy 2002;32(7):327-335. [PubMed: 12113467]

Lariviere C, Gagnon D, Loisel P. The effect of load on the coordination of the trunk for subjects with and without chronic low back pain during flexion-extension and lateral bending tasks. Clin Biomech (Bristol, Avon) 2000;15(6):407-416.

Marras WS, Lavender SA, Leurgans SE, Fathallah FA, Ferguson SA, Allread WG, et al. Biomechanical risk factors for occupationally related low back disorders. Ergonomics 1995;38(2):377-410. [PubMed: 7895740]

McGill SM. Low back stability: From formal description to issues for performance and rehabilitation. Exercise and Sport Sciences Reviews 2001;29(1):26-31. [PubMed: 11210443]

McGill SM, Kippers V. Transfer of loads between lumbar tissues during the flexion-relaxation phenomenon. Spine 1994;19(19):2190-2196. [PubMed: 7809753]

McGorry RW, Hsiang SM. A method for dynamic measurement of lumbar lordosis. J Spinal Disord 2000;13(2):118-123. [PubMed: 10780686]

Newcomer K, Laskowski ER, Yu B, Larson DR, An KN. Repositioning error in low back pain - comparing trunk repositioning error in subjects with chronic low back pain and control subjects. Spine 2000;25 (2):245-250. [PubMed: 10685490]

Olson MW, Li L, Solomonow M. Flexion-relaxation response to cyclic lumbar flexion. Clin Biomech (Bristol, Avon) 2004;19(8):769-776.

Panjabi MM. The stabilizing system of the spine. Part i. Function, dysfunction, adaptation, and enhancement. J Spinal Disord 1992a;5(4):383-389. [PubMed: 1490034]discussion 397

Panjabi MM. The stabilizing system of the spine. Part ii. Neutral zone and instability hypothesis. J Spinal Disord 1992b;5(4):390-396. [PubMed: 1490035]discussion 397

Panjabi MM. Clinical spinal instability and low back pain. J Electromyogr Kinesiol 2003;13(4):371-379. [PubMed: 12832167]

Punnett L, Fine LJ, Keyserling WM, Herrin GD, Chaffin DB. Back disorders and nonneutral trunk postures of automobile assembly workers. Scand J Work Environ Health 1991;17(5):337-346. [PubMed: 1835131] 
Roberts S, Eisenstein SM, Menage J, Evans EH, Ashton IK. Mechanoreceptors in intervertebral discs. Morphology, distribution, and neuropeptides. Spine 1995;20(24):2645-2651. [PubMed: 8747242]

Solomonow M. Ligaments: A source of work-related musculoskeletal disorders. J Electromyogr Kinesiol 2004;14(1):49-60. [PubMed: 14759750]

Solomonow M, Baratta RV, Banks A, Freudenberger C, Zhou BH. Flexion-relaxation response to static lumbar flexion in males and females. Clin Biomech (Bristol, Avon) 2003;18(4):273-279.

Solomonow M, Zhou BH, Harris M, Lu Y, Baratta RV. The ligamento-muscular stabilizing system of the spine. Spine 1998;23(23):2552-2562. [PubMed: 9854754]

Wilson SE, Granata KP. Reposition sense of lumbar curvature with flexed and asymmetric lifting postures. Spine 2003;28(5):513-518. [PubMed: 12616167] 


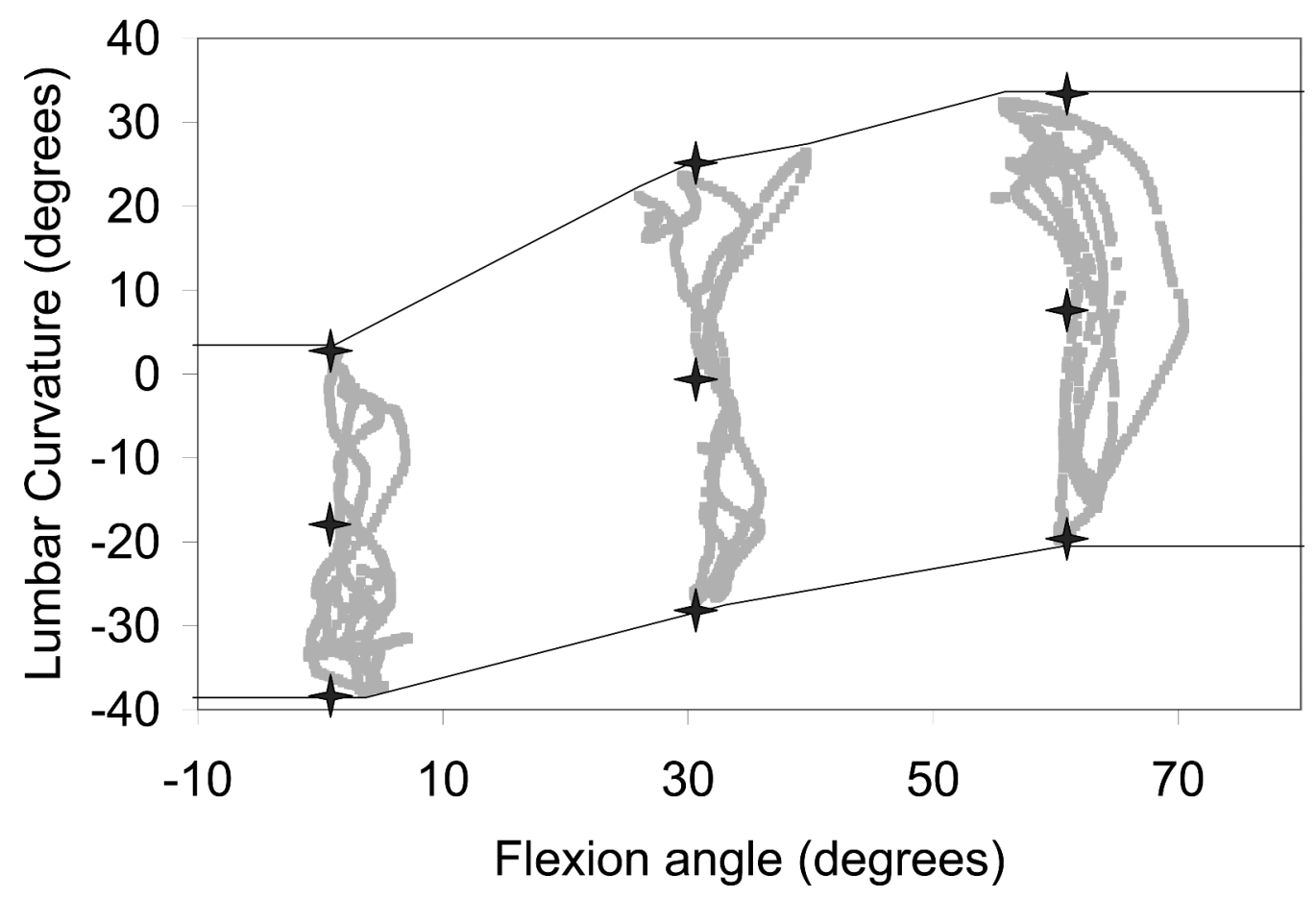

Figure 1.

This figure illustrates the range of lumbar curvatures that might be achievable by one subject at each of three torso flexion angles. By rotating their thorax and pelvis, subjects can alter their lumbar curvature while maintaining a given torso flexion angle. As torso flexion angle increases this range moves from more lordotic (negative) to more kyphotic (positive). For this experiment, target lumbar curvatures (stars) were chosen from the maximum and minimum lumbar curvature possible at a specified torso flexion angle. A third target lumbar curvature at the midpoint between the maximum and minimum curvature was also selected. 


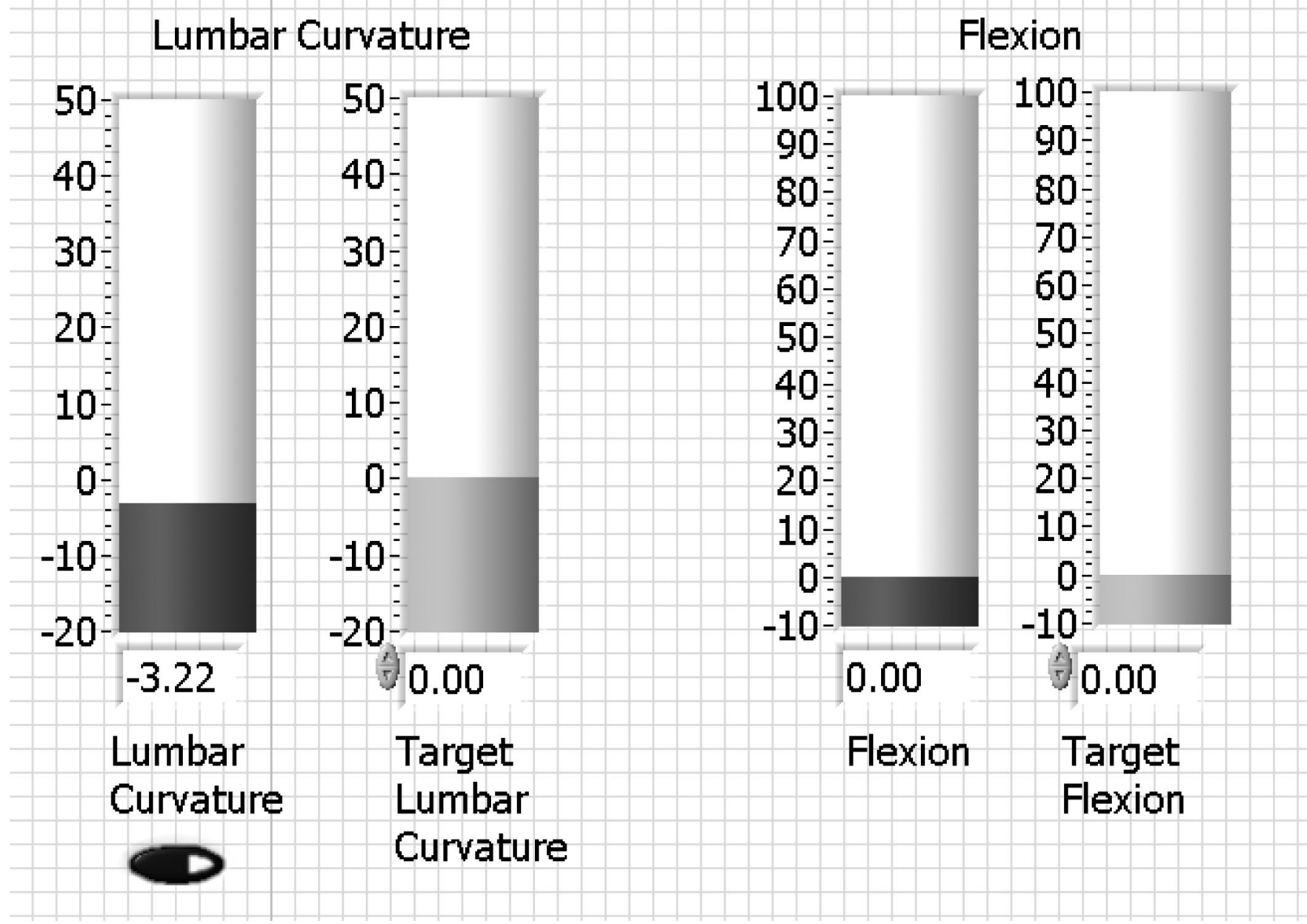

Figure 2.

A real-time visual display of torso flexion and lumbar curvature was provided for the subjects. Both torso flexion and lumbar curvature were displayed with two parts: the target posture that the subject should attempt to reach and the actual posture measured from the subject. Both a bar display and a large numeric display were available to the subjects. The bar displays were scaled to the range of the subjects motion. Subjects were asked to match the torso flexion display first and then match the lumbar display. In the assessment trials, the lumbar curvature display was turned off. 


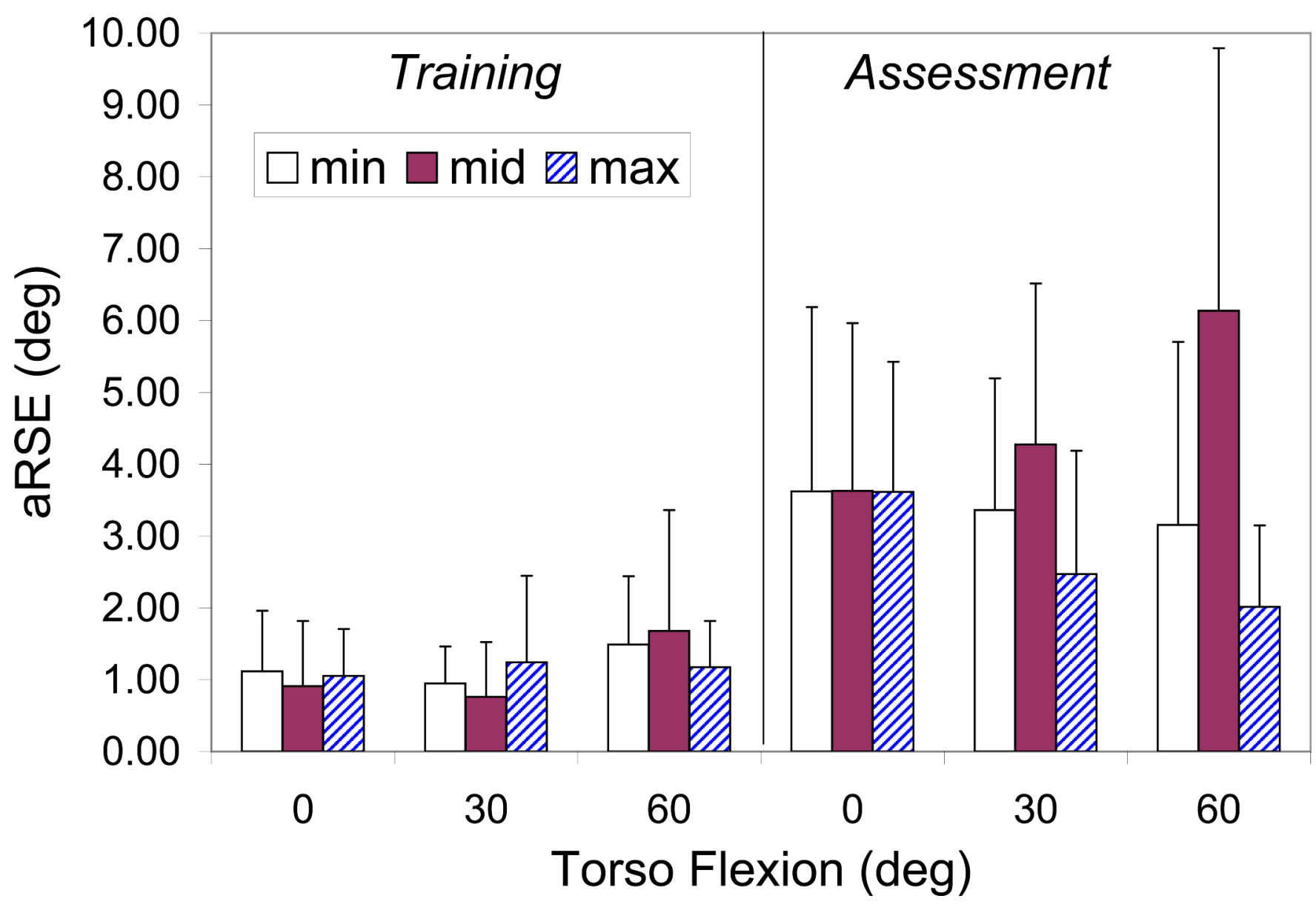

Figure 3.

Absolute reposition sense error (aRSE) was measured during both the training trials and the assessment trials. Absolute reposition sense error during assessment (aRSEa) increased with torso flexion for mid-range lumbar curvatures (means with standard deviation error bars are represented here). Both maximum and minimum lumbar curvature targets led to a reduction in the absolute reposition sense error from the mid-range. 


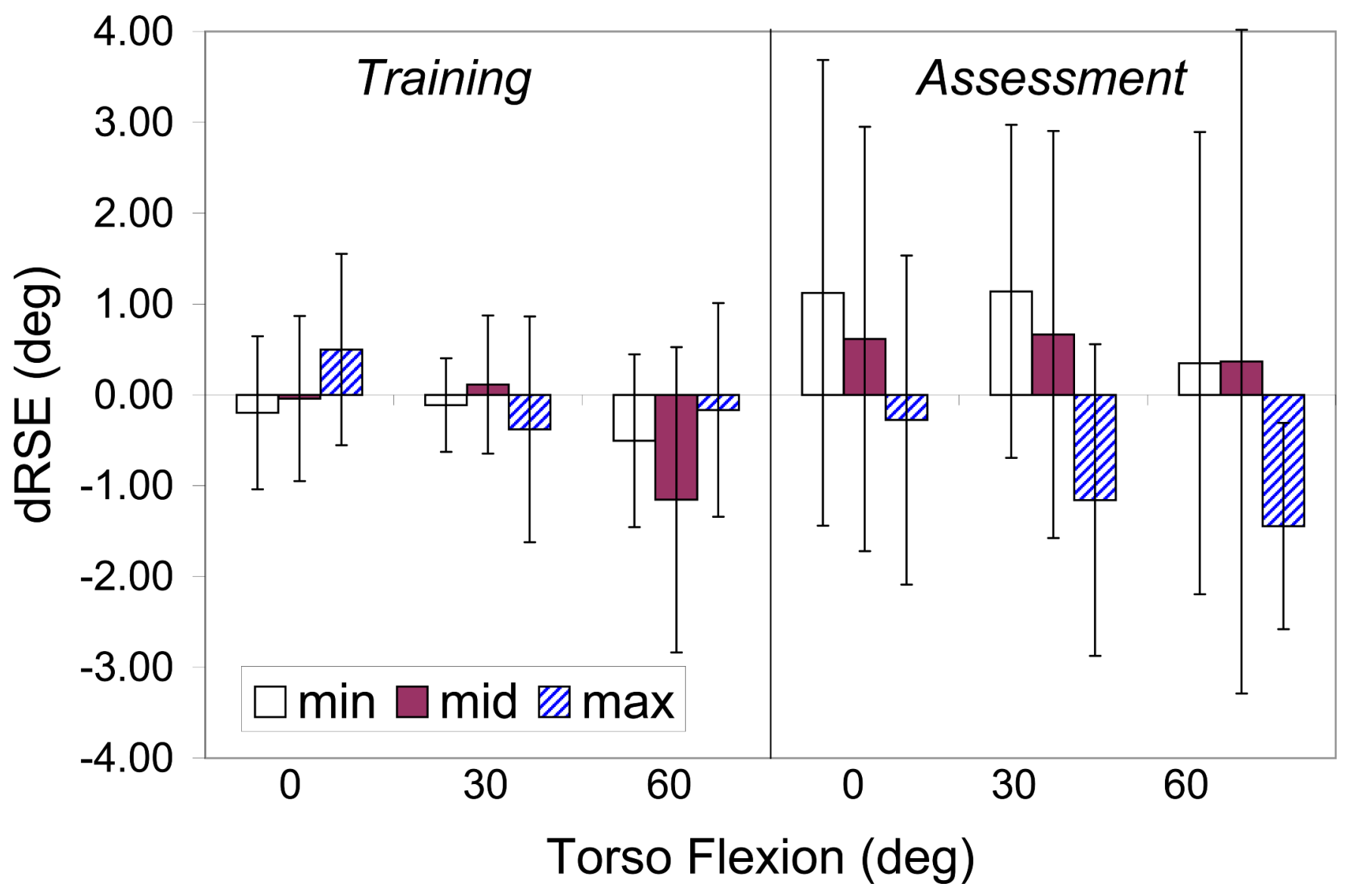

Figure 4.

Directional reposition sense errors (dRSE), representing the directional bias in position targeting, were small (means with standard deviation error bars are represented here). With maximum lumbar curvature, errors were negative and with minimum lumbar curvature, errors were positive. This indicates a small directional bias in the reposition errors towards a more neutral posture. 


\section{Table 1}

The minimum and maximum range of motion was assessed for each subject and the averages are displayed here (A). To examine the repeatability of the maximum and minimum lumbar angles at each torso flexion, the ranges of these measures averaged across all subjects is also reported (B).

\begin{tabular}{|l|r|r|}
\hline \multicolumn{3}{|c|}{ A. Average and Standard Deviation of the Lumbar Range } \\
\hline Torso Flexion & Minimum Lumbar Angle & Maximum Lumbar Angle \\
\hline 0 degrees & $-45.8 \pm 14.9 \mathrm{deg}$ & $3.1 \pm 18.8 \mathrm{deg}$ \\
\hline 30 degrees & $-26.1 \pm 18.0 \mathrm{deg}$ & $23.2 \pm 17.7 \mathrm{deg}$ \\
\hline 60 degrees & $0.4 \pm 21.6 \mathrm{deg}$ & $40.8 \pm 15.4 \mathrm{deg}$ \\
\hline
\end{tabular}

\begin{tabular}{|l|l|l|}
\hline \multicolumn{2}{|l|}{ B. Range of Lumbar Angle Measures (Average Across Subjects) } \\
\hline Torso Flexion & Minimum Lumbar Angle Range & Maximum Lumbar Angle Range \\
\hline 0 degrees & $9.6 \mathrm{deg}$ & $4.4 \mathrm{deg}$ \\
\hline 30 degrees & $6.3 \mathrm{deg}$ & $5.3 \mathrm{deg}$ \\
\hline 60 degrees & $8.3 \mathrm{deg}$ & $4.2 \mathrm{deg}$ \\
\hline
\end{tabular}




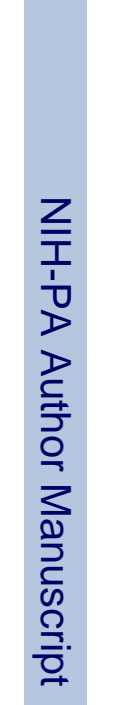

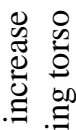

.

苛

壬 巳

3

के

के

.

F.

त)

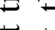

这

焉

证

政

○

蛋

要

壬 贯

i

$>\sim$

$>\div$

ल छ

음

ญ

등

ก के

무.

\begin{tabular}{|c|c|c|c|c|}
\hline & 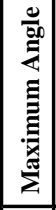 & 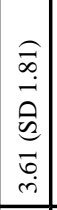 & 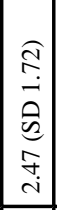 & 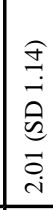 \\
\hline 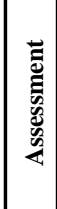 & 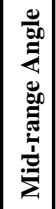 & 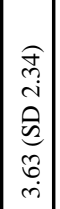 & 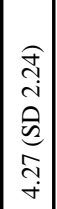 & 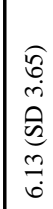 \\
\hline & 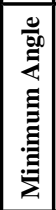 & 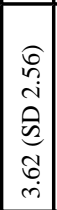 & 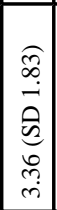 & 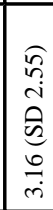 \\
\hline & 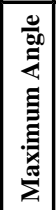 & $\begin{array}{l}\sigma \\
\sigma \\
0 \\
0 \\
0 \\
0 \\
0 \\
0 \\
-\end{array}$ & 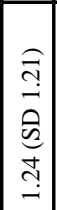 & $\mid \begin{array}{l}0 \\
6 \\
0 \\
0 \\
0 \\
0 \\
0\end{array}$ \\
\hline 音 & 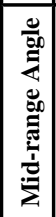 & $\mid \begin{array}{c}\hat{\imath} \\
0 \\
0 \\
\hat{0} \\
0 \\
\overrightarrow{0} \\
0\end{array}$ & $\mid$\begin{tabular}{c|c}
0 \\
0 \\
0 \\
0 \\
0 \\
0 \\
0 \\
0 \\
0 \\
0
\end{tabular} & 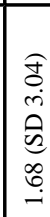 \\
\hline & 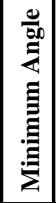 & 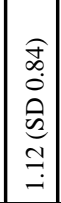 & $\mid \begin{array}{c}\hat{a} \\
0 \\
\vdots \\
\hat{0} \\
\vdots \\
\vdots \\
0 \\
0\end{array}$ & 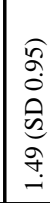 \\
\hline & 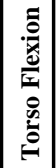 & 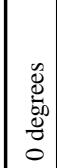 & 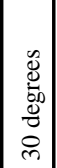 & \\
\hline
\end{tabular}

J Electromyogr Kinesiol. Author manuscript; available in PMC 2010 August 1. 


\section{Table 3}

A Huynh-Feldt, repeated measures ANOVA was used the examine the effects of the independent variables, torso flexion angle and lumbar angle, on the dependent variables, absolute reposition sense error (A) and directional reposition sense error (B) in the assessment trials.

\begin{tabular}{|c|c|c|c|c|c|c|c|c|}
\hline \multicolumn{9}{|c|}{ A. Absolute Reposition Sense Error (aRSEa) } \\
\hline \multicolumn{2}{|c|}{ Two-way ANOVA } & \multicolumn{2}{|c|}{ epsilon } & \multicolumn{2}{|r|}{ df } & \multicolumn{3}{|c|}{$\mathbf{F}$} \\
\hline \multicolumn{2}{|l|}{ Torso Flexion } & \multicolumn{2}{|c|}{0.909} & \multicolumn{2}{|c|}{1.819} & \multicolumn{2}{|c|}{0.315} & 0 . \\
\hline \multicolumn{2}{|l|}{ Lumbar Angle } & \multicolumn{2}{|c|}{0.872} & \multicolumn{2}{|c|}{1.744} & \multicolumn{2}{|c|}{13.025} & 0. \\
\hline \multicolumn{2}{|l|}{$T F * L C$} & \multicolumn{2}{|c|}{1.000} & \multicolumn{2}{|c|}{4.000} & \multicolumn{2}{|c|}{4.213} & 0. \\
\hline \multicolumn{9}{|c|}{ B. Directional Reposition Sense Error (dRSEa) } \\
\hline ANOVA & \multicolumn{2}{|c|}{ epsilon } & \multicolumn{2}{|r|}{ df } & \multicolumn{2}{|r|}{$\mathbf{F}$} & \multicolumn{2}{|r|}{$\mathbf{P}$} \\
\hline Torso Flexion & \multicolumn{2}{|c|}{0.931} & \multicolumn{2}{|c|}{1.862} & \multicolumn{2}{|c|}{0.647} & \multicolumn{2}{|c|}{0.521} \\
\hline Lumbar Angle & \multicolumn{2}{|c|}{1.000} & \multicolumn{2}{|c|}{2.000} & \multicolumn{2}{|c|}{3.989} & \multicolumn{2}{|c|}{0.030} \\
\hline $\mathrm{TF} * \mathrm{LC}$ & \multicolumn{2}{|c|}{0.911} & \multicolumn{2}{|c|}{3.643} & \multicolumn{2}{|c|}{0.177} & \multicolumn{2}{|c|}{0.938} \\
\hline
\end{tabular}




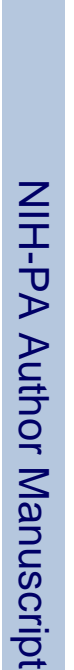

ㅇํ.

包

站 $\frac{0}{0}$

00

ส

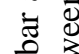

芯

छี

을

这

$\circ \frac{0}{2}$

을

¿

苍

.ำㅇำ옹

芯

.

巳

至 关

$i^{2}$

穸

$>$ 월

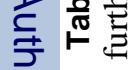

马

ญ 을

उั

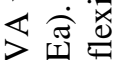

方至焉

\&

可 홍

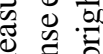

ఏ ‡

उृี

过 $\frac{0}{00}$

윤 辰

灾包

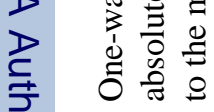

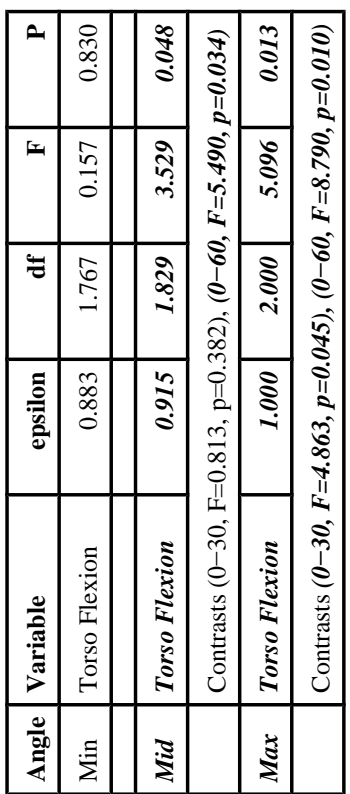

J Electromyogr Kinesiol. Author manuscript; available in PMC 2010 August 1. 


\section{Table 5}

Directional reposition sense error (degrees) remained close to zero during the training trials. During the assessment trials, this error was found to be negative with maximum lumbar angle targets and positive with minimum lumbar angle targets suggesting a directional bias towards a more neutral posture. For all data the standard deviation (SD) and 95\% confidence interval are reported.

\begin{tabular}{|l|c|c|c|}
\hline & \multicolumn{3}{|c|}{ Training } \\
\hline Torso Flexion & Minimum Lumbar Angle & Mid-range Lumbar Angle & Maximum Lumbar Angle \\
\hline 0 degrees & -0.20 (SD 1.23, CI -0.80 to 0.41$)$ & -0.04 (SD 1.11, CI -0.59 to 0.50$)$ & 0.50 (SD 0.83, CI 0.09 to 0.90) \\
\hline 30 degrees & -0.11 (SD 0.80, CI -0.50 to 0.28$)$ & 0.11 (SD 0.54, CI -0.15 to 0.38) & -0.38 (SD 0.91, CI -0.83 to 0.07) \\
\hline 60 degrees & -0.50 (SD 1.51, CI -1.24 to 0.23$)$ & -1.16 (SD 3.21, CI -2.72 to 0.42$)$ & -0.17 (SD 1.22, CI -0.76 to 0.43$)$ \\
\hline
\end{tabular}

\begin{tabular}{|l|c|c|c|}
\hline & \multicolumn{3}{|c|}{ Assessment } \\
\hline Torso Flexion & Minimum Lumbar Angle & Mid-range Lumbar Angle & Maximum Lumbar Angle \\
\hline 0 degrees & 1.12 (SD 3.32, CI -0.50 to 2.75) & 0.62 (SD 2.76, CI -0.74 to 1.97) & -0.28 (SD 2.95, CI -1.72 to 1.17) \\
\hline 30 degrees & 1.14 (SD 2.81, CI -0.23 to 2.51) & 0.66 (SD 2.66, CI -0.63 to 1.97) & -1.16 (SD 2.26, CI -2.27 to -0.05$)$ \\
\hline 60 degrees & 0.35 (SD 3.39, CI -1.31 to 2.01) & 0.37 (SD 4.90, CI -2.03 to 2.77) & -1.44 (SD 1.31, CI -2.09 to -0.80$)$ \\
\hline
\end{tabular}

